\title{
Use, perceptions, and acceptability of a ready-to- use supplementary food among adult HIV patients initiating antiretroviral treatment: a qualitative study in Ethiopia
}

\author{
This article was published in the following Dove Press journal: \\ Patient Preference and Adherence \\ 6 June 2013 \\ Number of times this article has been viewed
}

\section{Mette Frahm Olsen' \\ Markos Tesfaye ${ }^{2}$ \\ Pernille Kaestel' \\ Henrik Friis' \\ Lotte Holm ${ }^{3}$}

'Department of Nutrition, Exercise and Sports, Faculty of Science, University of Copenhagen, Frederiksberg, Denmark;

2Department of Psychiatry, College of Public Health and Medical Sciences, Jimma University, Jimma, Ethiopia; ${ }^{3}$ Department of Food and Resource Economics, Faculty of Science, University of Copenhagen, Frederiksberg, Denmark
Correspondence: Mette Frahm Olsen Department of Nutrition, Exercise and Sports, Faculty of Science, University of Copenhagen, Rolighedsvej 30, 1958 Frederiksberg C, Denmark

Tel +453533253 I

Fax +45 35332483

Email meo@life.ku.dk
Objectives: Ready-to-use supplementary foods (RUSF) are used increasingly in human immunodeficiency virus (HIV) programs, but little is known about how it is used and viewed by patients. We used qualitative methods to explore the use, perceptions, and acceptability of RUSF among adult HIV patients in Jimma, Ethiopia.

Methods: The study obtained data from direct observations and 24 in-depth interviews with HIV patients receiving RUSF.

Results: Participants were generally very motivated to take RUSF and viewed it as beneficial. RUSF was described as a means to fill a nutritional gap, to "rebuild the body," and protect it from harmful effects of antiretroviral treatment (ART). Many experienced nausea and vomiting when starting the supplement. This caused some to stop supplementation, but the majority adapted to RUSF. The supplement was eaten separately from meal situations and only had a little influence on household food practices. RUSF was described as food with "medicinal qualities," which meant that many social and religious conventions related to food did not apply to it. The main concerns about RUSF related to the risk of HIV disclosure and its social consequences.

Conclusion: HIV patients view RUSF in a context of competing livelihood needs. RUSF intake was motivated by a strong wish to get well, while the risk of HIV disclosure caused concerns. Despite the motivation for improving health, the preservation of social networks was prioritized, and nondisclosure was often a necessary strategy. Food sharing and religious fasting practices were not barriers to the acceptability of RUSF. This study highlights the importance of ensuring that supplementation strategies, like other HIV services, are compatible with the sociocultural context of patients.

Keywords: Africa, nutritional supplementation, qualitative research, adherence

\section{Introduction}

The human immunodeficiency virus (HIV) epidemic in sub-Saharan Africa is closely linked to food insecurity and malnutrition. ${ }^{1-3}$ Poor nutritional status at initiation of antiretroviral treatment (ART) is associated with adverse treatment outcomes and high mortality during the early phase of ART. ${ }^{4-8}$ Studies suggest that ready-to-use supplementary foods (RUSF), originally developed for children, can improve the treatment of adult HIV patients. ${ }^{9-11}$ The distribution of RUSF to patients has already become a widely used strategy among international organizations ${ }^{12,13}$ and national HIV programs in sub-Saharan Africa, ${ }^{14}$ but little is known about the use and perceptions of these supplements. So far, only a few studies have reported on the acceptance of RUSF among 
HIV patients. Poor adherence was reported from studies in Kenya ${ }^{15}$ and Malawi ${ }^{16}$ despite overall positive ratings of the supplement. In Kenya, patients reported the main barriers as nausea and vomiting in the first days of supplementation, unfamiliar taste and consistency, dietary boredom, and stigma as RUSF became associated with HIV. ${ }^{15}$

Since the rollout of ART, patient barriers for treatment uptake and adherence have been a topic of ongoing research. In this field, it has become increasingly clear that adherence is not just a matter of ability in terms of time and cost, but also of willingness to prioritize ART over competing livelihood needs. ${ }^{17,19}$ In a meta-ethnography, Merten et al described a strong motivation to follow treatment, driven by patients' wish to fulfill life ambitions and social responsibilities. However, stigma continues to be a main barrier to the willingness to take ART. Nondisclosure of HIV is often a necessary strategy to maintain social networks. The need for physical health must be balanced against the need for social inclusion, in settings where resources are scarce and formal social security is absent. If stigma is anticipated, HIV services that involve risk of disclosure are often avoided. ${ }^{17}$ Similar patient concerns must be considered when providing bulky portions of RUSF for home supplementation. In addition, the feasibility and acceptability of providing individual supplementation needs to be explored in settings were food is usually shared among family members and food insecurity is common. Furthermore, food intake is often affected by religious fasting practices. The social and cultural norms that pertain to food differ from those pertaining to medicine, ${ }^{18}$ emphasizing the importance of exploring whether RUSF is categorized as food or medicine by those receiving it.

In this study, we used qualitative methods to explore the use, perceptions, and acceptability of individual RUSF supplementation among Ethiopian HIV patients. Rather than seeking to quantify adherence, our aim was to gain insight to the participants' experience of RUSF, including their motivation and barriers for consumption, their acceptance of individual supplementation, and their rationales for sharing it or not.

\section{Methods}

The study was conducted at the Jimma University Specialized Hospital, as part of a nutrition supplementation trial in Jimma, Southwest Ethiopia. More than one million Ethiopians are living with HIV, which has a prevalence of $4.2 \%$ in urban and $0.6 \%$ in rural areas. ${ }^{20}$ Studies in Jimma have shown that HIV patients are often affected by food insecurity, ${ }^{21,22}$ although the area is generally considered food secure and is not included in the World Food Programme urban HIV food supplementation program. ${ }^{23,24}$

The trial investigated the effects of providing RUSF after initiation of ART on general and HIV-specific outcomes (registered at www.pactr.org [PACTR201110000330271] and www.controlled-trials.com [ISRCTN32453477]). Patients $\geq 18$ years who had BMI $\geq 16 \mathrm{~kg} / \mathrm{m}^{2}$ were invited to participate, while patients with BMI $<16 \mathrm{~kg} / \mathrm{m}^{2}$ were referred to standard care. ${ }^{14}$ Trial participants received $200 \mathrm{~g}$ RUSF per day $(\sim 1000 \mathrm{kcal})$ for 3 months. The RUSF product was developed by Nutriset SAS (Malaunay, France) and based on Plumpy'Sup ${ }^{\circledR}$ (Nutriset SAS), with $30 \mathrm{~g}$ /day of whey or soy protein. It was packaged in neutral sachets and provided in assorted backpacks.

A pretrial pilot test of the RUSF was conducted among 74 HIV patients receiving ART. The test included an evaluation of the taste and ranking of four flavor options (chocolate, banana, spicy, and plain peanut) as well as a 5-day test of the trial's ration. On completion, participants reported their consumption and overall perception of RUSF. The spicy flavor received the poorest rankings and was therefore not included in the trial. The remaining three flavor options were all included to reduce the risk of sensory boredom. Half of the pilot test participants found the daily amount was "more than sufficient", but still $90 \%$ of them found it would be feasible to consume this amount over a 3-months supplementation period. As well, non-sweet versions with tomato, onion, and spicy flavor, intended to be mixed with other foods, were tested. However, participants were reluctant to combine RUSF with their usual Ethiopian dishes of injera (fermented flatbread) and wat (sauces), and these were therefore not included in the trial.

Trial participants were offered a trial of the RUSF for 2 days before final enrolment. They were instructed not to share and to consume the RUSF without cooking. Adherence was assessed by counting the returned sachets monthly and by patients' reported RUSF intake over the previous 3 days. After 1 month of supplementation, the median intake (interquartile range [IQR]) was 176 (116-196) g/day, and 74\% reported to have consumed "everything" or "almost everything," while $22 \%$ reported to have consumed "nothing" or "only a little" over the preceding 3 days.

Subjects for in-depth interviews were selected by purposive sampling to reflect trial participants in terms of gender, age, socioeconomic status, and experience with RUSF. An interview guide was developed on the basis of findings from the pilot test, observations during the initial phase of the trial, and the authors' previous qualitative 
work in the setting. ${ }^{22}$ The guide was semistructured and explored the following topics: household food practices, the experience of HIV and ART initiation, use and perceptions of RUSF, and the acceptability of supplementation in relation to social, cultural, and religious practices and ideas. The interviewer asked open questions and invited participants to speak freely and in their own manner about the topics raised. The guide was piloted on the first four interviews, then reviewed and further adapted. The interviews took place in a quiet area within the clinic and were conducted in Amharic or Afaan Oromo, with the assistance of an experienced translator. The number of interviews was based on the principle of data saturation, including new participants as long as new themes were emerging. All interviews were recorded and transcribed in English. The data were analyzed according to the principles of qualitative content analysis discussed by Graneheim and Lundman. ${ }^{25}$ The procedure began with reading through all data several times to obtain a sense of the whole. The text was then divided into "meaning units" corresponding to full participant statements. These meaning units were condensed and labeled with codes. Codes where then compared and sorted into categories and thereafter, broader themes, in an iterative process moving between the whole and parts of the interview transcriptions. The broader context was considered in the interpretation of data, and in this process, deviant cases were searched for. Observation notes of nonverbal communication during interviews and of the team's daily communication with participants were also considered in this process. The findings and their implications were discussed with the study team.

Ethical permission for the study was obtained from the Ethiopian National Health Research Ethical Review Committee and Jimma University Ethical Review Board. A consultative approval was obtained from the Danish National Committee on Biomedical Research Ethics. Informed consent was obtained before the interviews, and all information was handled confidentially.

\section{Results}

\section{Participant characteristics}

A total of 24 in-depth interviews were conducted during the fall of 2010. The characteristics of the interviewed patients are presented in Table 1. Patients had been taking RUSF for 2 weeks (three patients), 1 month (two patients), 2 months (six patients), or 3 months (nine patients) at the time of interview. Additional interviews were conducted with patients who had discontinued RUSF during the first few
Table I Demographics of interviewees $(n=24)$

\begin{tabular}{ll}
\hline Female gender & $63 \%$ \\
Age & \\
Mean & 35 years \\
Range & $20-60$ years \\
Education & \\
No formal schooling & $25 \%$ \\
Primary school & $50 \%$ \\
Secondary school or higher & $25 \%$ \\
Religion & \\
Orthodox Christian & $67 \%$ \\
Muslim & $21 \%$ \\
Protestant & $13 \%$ \\
Marital status & \\
Married/cohabitating & $30 \%$ \\
Widower/divorced & $65 \%$ \\
Single, never married & $4 \%$ \\
Time taking RUSF when interviewed & \\
$<2$ weeks & $17 \%$ \\
2 weeks & $13 \%$ \\
I month & $8 \%$ \\
2 months & $25 \%$ \\
3 months & $38 \%$ \\
\hline
\end{tabular}

Abbreviation: RUSF, ready-to-use supplementary foods.

weeks of supplementation (four patients). The duration of the interviews was $1-1.5$ hours. Interview participants included 15 women and nine men, with age ranging between 20 and 60 years. The majority had no or only primary schooling. They were Orthodox Christians (67\%), Muslims (21\%), and Protestants (13\%), and two-thirds were living in singleheaded households (divorced or widowed). All patients approached agreed to participate.

\section{Motivation and barriers for taking RUSF}

Participants were generally pleased to receive RUSF and had high expectations of its effects. RUSF was described to "give energy and strength," "help the body rebuild," and "fill the blood." Many patients were concerned about losing weight and appearing ill. RUSF was said to "open" the appetite and help patients regain weight. As one woman said:

It is good food, and it is after I ate this food that I got my energy and gained weight. And also, my appetite has been opened.

There was a high awareness that HIV patients have increased nutritional needs. This caused frustration, as many found it difficult to obtain adequate food. Problems of food insecurity were often exacerbated by HIV. However, RUSF was described as a "complete food" and a substitute for a balanced diet. A male participant said: 
It's the only food I have. It's my meat, butter, and all the juices. Since I don't have the capacity to buy all those, the supplement is being all that for me.

A perception that "medicine requires food" was also expressed, and several participants said that RUSF would protect the body from the strong ART medicine. As a woman explained:

It helps me to take the medicine well. So that the medicine can keep well in my body.

In spite of the positive views, there were many accounts of nausea and vomiting when starting the supplement. Many were unable to consume the daily ration in the first few weeks of ART, when adverse effects were common. Participants had started ART and RUSF at the same time and were not able to distinguish which was causing discomfort. This made it a difficult time to introduce a supplement unfamiliar in taste and texture. A woman said:

At first I didn't like it. I wasn't accustomed to it, that's why.

It was the sweetness that I didn't like. There was nausea, and I didn't like it because I don't like sweets much.

Participants appreciated being able to choose between different flavors, but the sweetness was often described as "too much" and as making the supplement "heavy" to take.

The anticipation of health effects motivated the consumption of RUSF. A man said:

Yes, yes, because they have told me "you should finish" and because they are physicians and thinking that they know, I thought I have to finish; it is related with the medication.

Another man serves as an example of how hard some tried to overcome their discomfort:

I didn't hate eating the food. But when I swallowed it, it made me want to vomit. I finished the first pack, forcing myself. I also finished the second pack, forcing myself, and the same with the third one. After that, when I am near the smell of it, it simply disgusts me.

The participant decided to discontinue RUSF, but the majority said they slowly adapted to the new food.

The main concerns about RUSF regarded the risk of disclosure. Disclosure often led to serious consequences. Several participants said they had not been able to borrow food or money, or use common toilets and water taps after their HIV status was disclosed in their community. Most participants had only told close family members and worried about bringing home a bulky supplement. A woman working as a house servant said she had no privacy for taking RUSF:

I was worried and stressed about what they were going to give me.... The main thing I was worried about was where I was going to take the supplement, with me working at people's households.

Another participant told us she had lost her job as a servant, when the employer saw the supplement.

To avoid disclosure, RUSF was often hidden from visitors and even family members. Although provided in neutral and assorted backpacks, RUSF attracted attention and participants often had to invent "cover stories" about it. As a woman described:

My children didn't see it [the supplement]. I took it in a bag, and I told them it's for someone else. The youngest was at home, and she asked me where I got the bag from and said that she can use it for school. I told her no, it is for somebody, and I brought it to deliver to that person. And I locked it in, so she didn't see what was inside. None of them saw it.

Hiding the supplement was a very common strategy, while others told their family RUSF was a treatment for some other condition.

\section{Use of RUSF and household food practices}

The hiding and secrecy around RUSF meant that it did not become integrated with the usual food practices of participants. Most participants only consumed RUSF when they were alone and thereby kept it separate from both the usual food and meal situations of the household. It was not considered part of the household food and was mainly consumed between meals. Many had initially taken RUSF with bread or other foods, but generally, it was preferred to take it by itself directly from the sachet.

The daily ration was described as something that should be taken in addition to the usual food. A male participant said:

I am using the supplement as an additional food. I don't eat the supplement, stopping the other food that I eat.

However, food shortage would sometimes make participants rely on RUSF to substitute their intake of household food. A young woman explained:

The conditions gets tough at times and there are times where food will not be there. I think "What about them [her younger sisters] if I eat?" This feeling gets to me, and leaving what is there for them, I go out. 


\section{Sharing}

Food insecurity was common among the participants. Some received food assistance from local organizations consisting mainly of wheat flour and oil. Although targeted for the individual, these rations were usually included in the household food. In contrast, most participants explained that they did not share RUSF. Many completely avoided sharing by hiding the supplement and only eating it when alone. Some told their children that it was harmful for them. The fact that RUSF had been given specifically to HIV patients was a strong reason for not sharing it. Unlike food, medicine was not thought of as something to share. As a participant put it:

I have said it is medication, and medication cannot be used for two.

Many felt it was okay not to share because they saw RUSF as part of their treatment. A woman explained:

For it is my life, I will not pass my life to someone else.

No, it does not worry me much. I have not shared anything of it with any person.

The arguments for not sharing RUSF were often based on the fact that it was intended for "specific needs of HIV patients." As a woman said:

It's the right thing to do. If those people are healthy, then they don't need it as much as I need it. This is for me - to help me, right?

There were only few examples of participants who said they had shared RUSF, but several described situations where they would have liked to share. A woman became very emotional, when she explained how difficult it was not to share with her daughter:

In my heart I always say that it would have been great sharing and eating together. But they told me firmly that I should not share it, so I don't share it with anybody.

It was described as especially difficult not to share with children. A participant told her husband not to eat RUSF, but she said that telling a child would have been impossible:

In fact if it had been children, they would eat, but he is an adult. If it were little children, I would be forced to give.

Illness was another reason for sharing. A woman with three children shared RUSF with her HIV positive son:

You know it's a mother's heart. Especially for my son who is sick, I gave him two packs thinking that it might help him. ... But for my other children, I didn't give them. ... I told them that it would kill them if they eat it.

\section{Breaking the fast}

The majority of participants were Orthodox Christian. Members of the Ethiopian Orthodox Church fast up to 180 days a year, including every Wednesday and Friday. Fasting involves avoidance of all animal products, but the whey content of RUSF was not a concern to any of the participants in the study. Many explained that, because of HIV, they gave priority to their health above religious practices. A woman said:

There is no problem for me. Ever since I got the virus in my

body, I have decided to eat and live. So I stopped fasting

and started eating.

Some had discussed the issue with their priest and found that RUSF was accepted because it was given for HIV. Similarly, Muslim participants said they would break the fast during Ramadan if it benefitted them in relation to their health. In line with the sharing issues, it appeared that there were different rules for people who were ill. Getting well had become the main priority.

\section{Food or medicine?}

As shown above, RUSF was alternately described with the characteristics of food or medicine depending on the situations discussed during interviews. The supplement was seen as food because it satisfied the appetite, and participants felt it made them gain weight. But at the same time, RUSF was described as medicine because it was provided at the hospital, given specifically for HIV, and associated with the treatment. Thereby, RUSF was in a category of its own. A female participant said:

After I got used to it, I know its benefits. My body changed, my energy changed, my food appetite changed. I know it has benefits. I know it has medicinal quality.

These medicinal qualities exempted RUSF from the social norms related to sharing and fasting practices.

\section{Discussion}

Although originally developed for children, RUSF products are being used in an increasing range of nutritional supplementation programs. This study contributes to a better understanding of the context that in which RUSF is used by those receiving it. We found that adult HIV patients were very motivated to consume RUSF and viewed this as beneficial. They felt it was filling a gap in their diet and that it protected them against adverse effects of ART. Despite these positive perceptions, nausea made intake of RUSF difficult in the 
beginning of supplementation. The risk of HIV disclosure was also a major concern to patients, while fasting and sharing practices were not barriers to supplementation.

The validity of information about adherence and sharing practices is difficult to ensure, and some degree of socially desirable response bias should be expected in this study. To limit this, the researchers conducting interviews in this study were not involved in administering RUSF. Nevertheless, the perceptions expressed by patients are, to some extent, a reflection of the counseling and information provided by the study team. Negative perceptions might have been understated and sharing perhaps more common than was reported to us. However, the qualitative approach of this study had the advantage of giving a more nuanced perspective of participants' ideas and concerns about RUSF than could have been obtained by an objective adherence assessment. Further study limitations include the small number of participants and the fact that we were unable to include patients with BMI $<16 \mathrm{~kg} / \mathrm{m}^{2}$. Also, the translation of data involved a potential loss of meaning. A central issue when discussing the validity of findings in qualitative analyses, is that the data always allows multiple interpretations. ${ }^{25}$ In this study, the authors' experience from previous work in the setting and from conducting pilot tests strengthened the validity of interpretation. Validity was further strengthened by including observation notes in the analyses of data.

Similar to the findings from Kenya, ${ }^{15,26}$ we found that patients reported complaints about the unfamiliar taste of RUSF. In Kenya, it was common to mix RUSF with local foods to overcome unwanted taste and dietary monotony. The patients in the present study appreciated having flavor alternatives, but mixing RUSF with other food was not popular. Instead, patients generally chose to keep RUSF separate from their usual meal practices. Mixing RUSF with household food makes it difficult to hide and perhaps also more difficult not to share. In fact, sharing was reported as common in Kenya, in contrast to this study. While we found food donated by organizations was widely shared, RUSF was less likely to be so. In line with this, a Malawian study found that participants shared RUSF less frequently than a corn-soy blend that held closer resemblance to the usual diet. ${ }^{9}$

Sharing is a concern in any nutritional supplementation program, and it is widely recognized that sharing practices are difficult to avoid. Just as mothers are unlikely to withhold food from their children, individuals in highly food-insecure households are unlikely to refuse sharing. It has therefore been suggested that HIV patients cannot be expected to reserve food rations for themselves. ${ }^{12}$ However, this study shows that an association between RUSF and medicine sets aside many of the cultural and social norms pertaining to food. The individual receiving RUSF is seen as a patient under treatment. Thereby, the person takes on a social "sick role" that has been described by Parsons as a state of "sanctioned deviance," where the patient holds special rights and obligations, including the right to be exempted from social norms. ${ }^{27}$

In line with studies of ART uptake and adherence, we found that the risk of disclosing HIV status was a major concern to patients. The participation in and adherence to any HIV service are weighed against competing needs that might be jeopardized by disclosure. ${ }^{17}$ In a previous study from Jimma, we described that HIV disclosure led to loss of job, house eviction, or being cut off from usual coping practices of borrowing food or money in the community in times of shortfall. ${ }^{22}$ It is therefore not surprising that many participants choose not to talk openly about HIV. A survey showed that only $7 \%$ of patients in Jimma had disclosed their illness to neighbors and only $6 \%$ to their children, ${ }^{28}$ and a qualitative study found that nondisclosure was a main barrier to ART adherence. ${ }^{29}$ To avoid disclosure, participants in this study were hiding RUSF or making up alternative stories about its purpose. Similarly, stigma quickly became related with Plumpy'Nut ${ }^{\circledR}$ given to HIV patients in Kenya. ${ }^{15}$

Stigma should ideally be addressed and disclosure promoted in HIV programming, but the reality faced by many patients is that they cannot afford to live openly with HIV. Planners of nutritional support strategies must therefore recognize the concerns of patients and try to minimize the risk of disclosure that home supplementation poses.

Nausea and vomiting were common complaints of participants in this study as well as in studies in Kenya. ${ }^{15,26} \mathrm{We}$ found that some participants forced the consumption of RUSF and developed strong aversions to it. Patients receiving RUSF should be informed about the risk of adverse effects and advised to increase their consumption only as much as they can tolerate. Future studies of RUSF would be strengthened by a thorough registration of adverse effects among HIV patients.

Although not the focus of this study, our findings also bring attention to the challenges of household food insecurity that affect HIV patients. RUSF is a time-limited strategy targeting the elevated nutritional needs induced by ART, but participants tended to view it as a means to obtain an adequate diet. This observation highlights the continued need for other types of interventions. Different approaches are needed to target both the elevated nutritional needs induced by HIV and the broader scale food security issues that affect patients and their families.

The participants of this study live in a context of urban poverty and food insecurity, and their challenges are shared 
with many HIV patients in low-income countries. The findings presented here therefore have relevance for other African settings. However, food practices and perceptions are closely intertwined with culture. Therefore, a key point of this paper is that the specific sociocultural context of HIV patients should be considered in each specific setting before introducing a new nutritional supplement.

\section{Acknowledgments}

This study was conducted within the framework of a larger intervention study, ARTfood, based on funding from USDEC and with RUSF partly donated by Nutriset. The authors would like to acknowledge the efforts of the study team and, in particular, the participants of ARTfood.

\section{Disclosure}

The funders had no role in study design, data collection and analysis, the decision to publish, or preparation of the manuscript. The authors report no conflicts of interest in this work.

\section{References}

1. Anabwani G, Navario P. Nutrition and HIV/AIDS in sub-Saharan Africa: an overview. Nutrition. 2005;21(1):96-99.

2. Anema A, Vogenthaler N, Frongillo EA, Kadiyala S, Weiser SD. Food insecurity and HIV/AIDS: current knowledge, gaps, and research priorities. Curr HIV/AIDS Rep. 2009;6(4):224-231.

3. De Pee S, Semba RD. Role of nutrition in HIV infection: review of evidence for more effective programming in resource-limited settings. Food Nutr Bull. 2010;31(4):S313-S344.

4. Lawn SD, Harries AD, Anglaret X, Myer L, Wood R. Early mortality among adults accessing antiretroviral treatment programmes in subSaharan Africa. AIDS. 2008;22(15):1897-1908.

5. Paton NI, Sangeetha S, Earnest A, Bellamy R. The impact of malnutrition on survival and the CD4 count response in HIV-infected patients starting antiretroviral therapy. HIV Med. 2006;7(5):323-330.

6. Moh R, Danel C, Messou E, et al. Incidence and determinants of mortality and morbidity following early antiretroviral therapy initiation in HIVinfected adults in West Africa. AIDS. 2007;21(18):2483-2491.

7. May M, Boulle A, Phiri S, et al; IeDEA Southern Africa and West Africa. Prognosis of patients with HIV-1 infection starting antiretroviral therapy in sub-Saharan Africa: a collaborative analysis of scale-up programmes. Lancet. 2010;376(9739):449-457.

8. Zachariah R, Harries K, Moses M, et al. Very early mortality in patients starting antiretroviral treatment at primary health centres in rural Malawi. Trop Med Int Health. 2009;14(7):713-721.

9. Ndekha MJ, van Oosterhout JJ, Zijlstra EE, Manary M, Saloojee H, Manary MJ. Supplementary feeding with either ready-to-use fortified spread or corn-soy blend in wasted adults starting antiretroviral therapy in Malawi: randomised, investigator blinded, controlled trial. BMJ. 2009;338:b1867.

10. van Oosterhout JJ, Ndekha M, Moore E, Kumwenda JJ, Zijlstra EE, Manary M. The benefit of supplementary feeding for wasted Malawian adults initiating ART. AIDS Care. 2010;22(6):737-742.

11. Ahoua L, Umutoni C, Huerga H, et al. Nutrition outcomes of HIV-infected malnourished adults treated with ready-to-use therapeutic food in subSaharan Africa: a longitudinal study. J Int AIDS Soc. 2011;14:2.
12. Webb P, Rogers B, Rosenberg I, et al. Improving the Nutritional Quality of US Food Aid: Recommendations for Changes to Products and Programs. Boston MA: Tufts University; 2011. Available from: http:// nutrition.tufts.edu/news/food-aid-quality-report. Accessed May 6, 2013.

13. Chaparro CM, Dewey KG. Use of lipid-based nutrient supplements (LNS) to improve the nutrient adequacy of general food distribution rations for vulnerable sub-groups in emergency settings. Matern Child Nutr. 2010;6 Suppl 1:1-69.

14. Federal Ministry of Health. National Guidelines for HIV/AIDS and Nutrition in Ethiopia. Addis Ababa: The Federal Democratic Republic of Ethiopia Ministry of Health; 2008.

15. Dibari F, Bahwere P, Le Gall I, Guerrero S, Mwaniki D, Seal A. A qualitative investigation of adherence to nutritional therapy in malnourished adult AIDS patients in Kenya. Public Health Nutr. 2012;15(2):316-323.

16. Bahwere P, Sadler K, Collins S. Acceptability and effectiveness of chickpea sesame-based ready-to-use therapeutic food in malnourished HIV-positive adults. Patient Prefer Adherence. 2009;3:67-75.

17. Merten S, Kenter E, McKenzie O, Musheke M, Ntalasha H, Martin-Hilber A. Patient-reported barriers and drivers of adherence to antiretrovirals in sub-Saharan Africa: a meta-ethnography. Trop Med Int Health. 2010;15 Suppl 1:16-33.

18. Geest S van der, Whyte SR. The Context of Medicines in Developing Countries: Studies in Pharmaceutical Anthropology. Het Spinhuis; 1991.

19. Parkes-Ratanshi R, Bufumbo L, Nyanzi-Wakholi B, et al. Barriers to starting ART and how they can be overcome: individual and operational factors associated with early and late start of treatment. Trop Med Int Health. 2010;15(11):1347-1356.

20. Central Statistical Agency [Ethiopia], ICF International. Ethiopia Demographic and Health Survey 2011. Addis Ababa and Calverton, MD: Central Statistical Agency and ICF International; 2012. Available from: http://www.measuredhs.com/pubs/pdf/FR255/FR255.pdf. Accessed May 7, 2013.

21. Tiyou A, Belachew T, Alemseged F, Biadgilign S. Food insecurity and associated factors among HIV-infected individuals receiving highly active antiretroviral therapy in Jimma zone Southwest Ethiopia. Nutr J . 2012;11:51.

22. Olsen M, Jensen NK, Tesfaye M, Holm L. Conceptual equivalence of WHOQOL-HIV among people living with HIV in Ethiopia. Qual Life Res. 2013;22(2):361-367.

23. World Food Programme. Food Security Situation in Ethiopia. Special issue April 8, 2011. Rome, Italy: World Food Programme; 2011. Available from: http://www.wfp.org/content/ethiopia-food-securitysituation-2011. Accessed May 7, 2013.

24. World Food Programme. Ethiopia's Urban HIV/AIDS programme. Fact sheet. Rome: World Food Programme 2011. Available from: http://www. wfp.org/sites/default/files/Urban\%20HIV\%20AIDS.pdf

25. Graneheim UH, Lundman B. Qualitative content analysis in nursing research: concepts, procedures and measures to achieve trustworthiness. Nurse Educ Today. 2004;24(2):105-112.

26. Dibari F, Bahwere P, Huerga H, et al. Development of a cross-over randomized trial method to determine the acceptability and safety of novel ready-to-use therapeutic foods. Nutrition. 2013;29(1): 107-112.

27. Parsons T. The sick role and the role of the physician reconsidered. Milbank Mem Fund Q Health Soc. 1975;53(3):257-278.

28. Deribe K, Woldemichael K, Wondafrash M, Haile A, Amberbir A. Disclosure experience and associated factors among HIV positive men and women clinical service users in southwest Ethiopia. BMC Public Health. 2008;8:81.

29. Balcha TT, Jeppsson A, Bekele A. Barriers to antiretroviral treatment in Ethiopia: a qualitative study. J Int Assoc Physicians in AIDS Care (Chic). 2011;10(2):119-125. 


\section{Publish your work in this journal}

Patient Preference and Adherence is an international, peer-reviewed, open access journal focusing on the growing importance of patient preference and adherence throughout the therapeutic continuum. Patient satisfaction, acceptability, quality of life, compliance, persistence and their role in developing new therapeutic modalities and compounds to

optimize clinical outcomes for existing disease states are major areas of interest. This journal has been accepted for indexing on PubMed Central. The manuscript management system is completely online and includes a very quick and fair peer-review system. Visit http://www.dovepress.com/ testimonials.php to read real quotes from published authors.

Submit your manuscript here: http://www.dovepress.com/patient-preference-and-adherence-journal 